\title{
The euchromatic histone mark H3K36me3 preserves heterochromatin through sequestration of an acetyltransferase complex in fission yeast
}

\author{
Paula R. Georgescu ${ }^{1}$, Matías Capella ${ }^{1}$, Sabine Fischer-Burkart ${ }^{1}$ and Sigurd Braun ${ }^{1, *}$ \\ ${ }^{1}$ Department of Physiological Chemistry, BioMedical Center (BMC), Ludwig-Maximilians-Universität München, Martinsried, \\ Germany. \\ * Corresponding Author: \\ Sigurd Braun: Tel: +49 (89) 2180-77128; E-mail: Sigurd.Braun@bmc.med.Imu.de
}

ABSTRACT Maintaining the identity of chromatin states requires mechanisms that ensure their structural integrity through the concerted actions of histone modifiers, readers, and erasers. Histone H3K9me and H3K27me are hallmarks of repressed heterochromatin, whereas H3K4me and H3K36me are associated with actively transcribed euchromatin. Paradoxically, several studies have reported that loss of Set2, the methyltransferase responsible for H3K36me, causes de-repression of heterochromatin. Here we show that unconstrained activity of the acetyltransferase complex Mst2C, which antagonizes heterochromatin, is the main cause of the silencing defects observed in Set2-deficient cells. As previously shown, Mst2C is sequestered to actively transcribed chromatin via binding to $\mathrm{H} 3 \mathrm{~K} 36 \mathrm{me} 3$ that is recognized by the PWWP domain protein Pdp3. We demonstrate that combining deletions of $\operatorname{set}^{+}$and $p d p 3^{+}$results in an epistatic silencing phenotype. In contrast, deleting mst2 $^{+}$, or other members of Mst2C, fully restores silencing in Set2-deficient cells. Suppression of the silencing defect in set $2 \Delta$ cells is specific for pericentromeres and subtelomeres, which are marked by H3K9me, but is not seen for loci that lack genuine heterochromatin. Mst2 is known to acetylate histone H3K14 redundantly with the HAT Gnc5. Further, it is involved in the acetylation of the non-histone substrate and E3 ubiquitin ligase Brl1, resulting in increased H2B-K119 ubiquitylation at euchromatin. However, we reveal that none of these mechanisms are responsible for the Set2-dependent silencing pathway, implying that Mst2 targets another, unknown substrate critical for heterochromatin silencing. Our findings demonstrate that maintenance of chromatin states requires spatial constraint of opposing chromatin activities. doi: 10.15698/mic2020.03.711 Received originally: 19.08.2019; in revised form: 18.12.2019, Accepted 20.12.2019, Published 03.01.2020.

Keywords: chromatin, heterochromatin, silencing, acety/transferase, histone modification.
Abbreviations:
5-FOA - 5'-fluoroorotic acid, ChIP - chromatin immunoprecipitation, CTD - C-terminal domain, HAT - histone acetyltransferase, $H D A C$ - histone deacetylase, HULC - histone ubiquitin E3 ligase complex, $K A T-$ lysine acetyt/transferase, Mst2C-Mst2 complex, qPCR - quantitative $P C R$, RNAi - RNA interference, RNAP - RNA polymerase, SAGA - Spt-Ada-Gcn5 acetyltransferase, SHREC - Snf2/Hdac repressive complex, TAS - telomere-associated sequence, TERRA - telomeric repeat-containing non-coding RNA, WT-wild-type.

\section{INTRODUCTION}

The nucleus of eukaryotic cells is organized into topologically distinct chromatin domains, known as eu- and heterochromatin. Both are controlled through various posttranslational histone modifications, nucleosome remodeling and RNA-related processes. Euchromatin is associated with active transcription and histone hyperacetylation, contributing to an open chromatin structure. In contrast, heterochromatin is associated with gene repression and histone hypoacetylation, often adopting a compact chro- matin structure that restricts transcription and genomic recombination. Whereas constitutive heterochromatin is present at repeat-rich sequences like centromeres and telomeres, facultative heterochromatin can also form at gene-rich regions, e.g. during cellular differentiation and adaptation to environmental changes. Responding to these changes and maintaining the structural integrity of heterochromatin domains requires the concerted actions of histone modifiers, readers, and erasers [1-3]. 
A conserved type of heterochromatin is characterized by the presence of methylated lysine 9 of histone $\mathrm{H} 3$ (H3K9me) [2]. In Schizosaccharomyces pombe, distinct heterochromatin domains are present at pericentromeres, subtelomeres and the silent mating type locus. H3K9me is deposited by the sole histone methyltransferase Clr4 that is present in a complex known as CLRC (Clr4 methyltransferase complex) and catalyzes all three steps of methylation [4-6]. The repressive H3K9me mark is recognized by chromodomain-containing proteins, such as Clr4 itself [7] and members of the HP1 family that interact with various chromatin factors [8, 9]. Among those is the repressor complex SHREC (Snf2/Hdac repressive complex) that deacetylates histone $\mathrm{H} 3$ at lysine $\mathrm{K} 14$ (H3K14ac) and restricts access of RNA polymerase II (RNAPII) to heterochromatin [10-12].

Heterochromatin assembly is guided by several targeting mechanisms among which the RNA interference machinery plays a prominent role in $S$. pombe $[13,14]$. Conversely, euchromatin is protected from ectopic heterochromatin assembly by several heterochromatinantagonizing factors. The JmjC protein Epe1 counteracts H3K9me formation at euchromatic sites prone to heterochromatin assembly [15-17] and prevents spreading beyond heterochromatin boundaries [18]. Epe1 is recruited to HP1 proteins and competes with SHREC for HP1 binding, thereby facilitating access of RNAPII to chromatin [19-21]. Heterochromatin is further antagonized by the RNA polymerase RNAPII-associated factor 1 complex (Paf1C), which is involved in multiple steps in transcription. Mutants of Paf1C are susceptible to small interfering RNA (siRNA)mediated heterochromatin initiation at ectopic sites, possibly due to altered kinetics in the processing and termination of nascent transcripts [22-24]. Paf1C also affects heterochromatin maintenance through its subunit Leo1, which prevents spreading at heterochromatin boundaries and promotes histone turnover $[25,26]$. Furthermore, Paf1C's elongation function may help to overcome the repressive activity of $\mathrm{H} 3 \mathrm{~K} 9 \mathrm{me} 3$ by supporting RNAPII in disrupting nucleosomes [27].

Histone acetyltransferases (HATs) also counteract heterochromatin by altering the charge and structure of nucleosomes, and also through the recruitment of factors to acetylated histones. The lysine acetytltransferase (KAT) Mst2 mediates H3K14 acetylation redundantly with the HAT Gcn5, which is part of the SAGA (Spt-Ada-Gcn5 acetyltransferase) complex [28]. Loss of Mst2 enhances silencing at subtelomeres [29] and bypasses the need for RNA interference (RNAi) in centromeric heterochromatin maintenance [30]. Furthermore, the rate at which ectopic silencing is initiated in a paf1 mutant is drastically increased when Mst2 is absent [31]. Mst2 is present in a complex (Mst2C) homologous to Saccharomyces cerevisiae NuA3b, which contains the PWWP domain protein Pdp3 [28, 32]. Pdp3 binds to trimethylated H3K36 (H3K36me3) and sequesters Mst2 to actively transcribed chromatin [31, 32]. Notably, in Pdp3-deficient cells, Mst2 gains promiscuous access to heterochromatin, where it triggers a silencing defect [31]. However, none of these heterochromatin- associated phenotypes are recapitulated by the loss of Gcn5, implying that Mst2 has another, non-redundant function that involves an acetylation substrate other than H3K14 [30, 31]. Proteome analysis revealed that Mst2 is involved in the acetylation of Brl1, which is part of the histone ubiquitin E3 ligase complex (HULC). However, whether Brl1 acetylation is also responsible for the silencing defect under conditions when Mst2 encroaches on heterochromatin (i.e. in $p d p 3 \Delta$ cells) remains unknown.

H3K36 methylation is associated with actively transcribed chromatin. In budding and fission yeast, all three methylation states are mediated by a single enzyme, Set 2 [33]. Set2 binds to the phosphorylated C-terminal domain (CTD) of transcribing RNAPII through its Set2 Rpb1 interacting (SRI) domain, which is a prerequisite for $\mathrm{H} 3 \mathrm{~K} 36$ tri- but not dimethylation $[34,35]$. While H3K36 methylation is coupled to transcriptional elongation, it is also implicated in gene repression. In budding yeast, the histone deacetylase (HDAC) complex Rpd3S promotes histone deacetylation in the wake of transcribing RNAPII, which prevents initiation of aberrant transcription from cryptic promoters within coding regions. Rdp3S is recruited to chromatin through its interaction with the phosphorylated CTD of RNAPII [36]. In addition, the chromodomain subunits Eaf3 and Rco1 recognize di- and trimethylated H3K36 [37-40], which stimulates the HDAC activity of Rpd3S [36]. The fission yeast homologs of Rpd3S and Eaf3 are Clr6 complex II (Clr6C-II) and Alp13, respectively [41]. Mutants deficient in Set2 and Alp13 display increased antisense transcription in coding regions [41] and silencing defects at various heterochromatin domains $[34,42,43]$. Set2 is further required for the repression of subtelomeric regions, characterized by highly condensed chromatin bodies termed 'knobs', which lack H3K9me and most other histone modifications [44]. However, whether silencing defects in set $2 \Delta$ cells are mediated directly through a local loss of H3K36me or an alternative mechanism remains unclear.

Here we demonstrate that the silencing defects in set $2 \Delta$ cells at canonical heterochromatin can be fully reversed by concomitant deletion of $m s t 2^{+}$. Full suppression of the silencing defect is also seen in the absence of other Mst2C members, which are critical for proper complex assembly, but not for Pdp3 that recruits Mst2C to actively transcribed regions via H3K36me3. Together, our findings implicate that the silencing defect of set $2 \Delta$ cells is caused by the global mislocalization of Mst2C and its encroachment on heterochromatic regions.

\section{RESULTS \\ Deletion of the mst2 $^{+}$gene suppresses the silencing defect of Set2-decifienct cells}

We previously showed that loss of the PWWP subunit Pdp3 causes moderate silencing defects of the pericentromeric imr1L::ura $4^{+}$reporter gene and various subtelomeric genes, which are suppressed when $\mathrm{mst}^{+}$is concomitantly deleted [31]. Since Pdp3 anchors Mst2 to euchromatin via H3K36me3 [31], we tested whether the silencing defects of set $2 \Delta$ at various pericentromeric and subtelomeric hetero- 
A

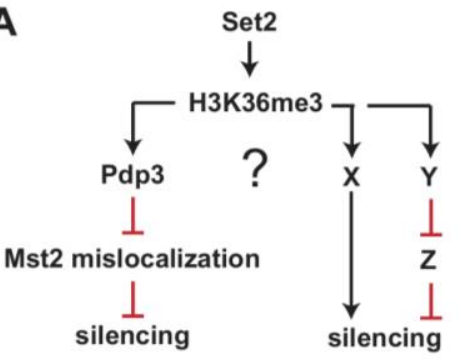

\section{RT-qPCR pericentromeres}
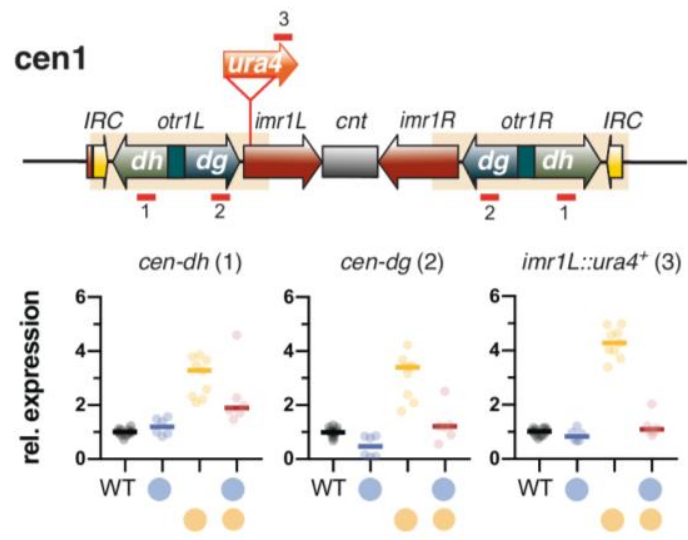

D ChIP-qPCR pericentromeres
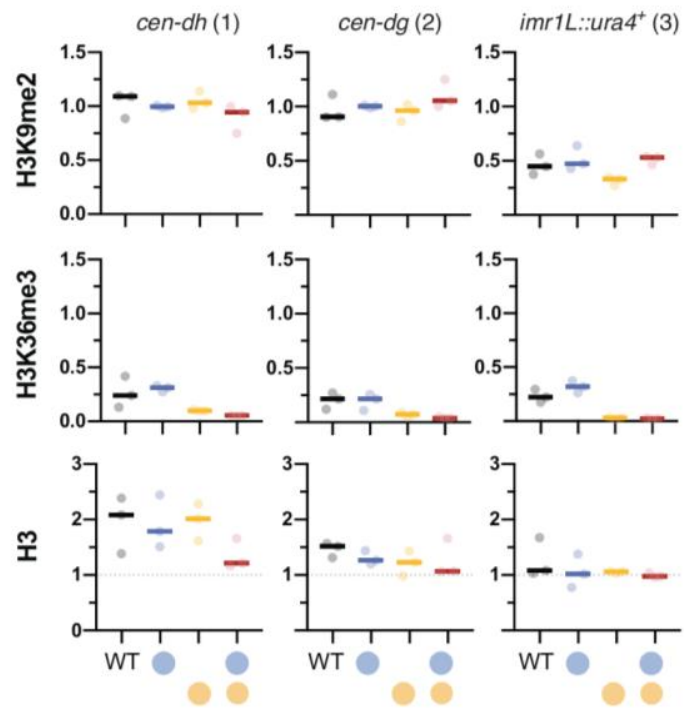

B Silencing reporter assay

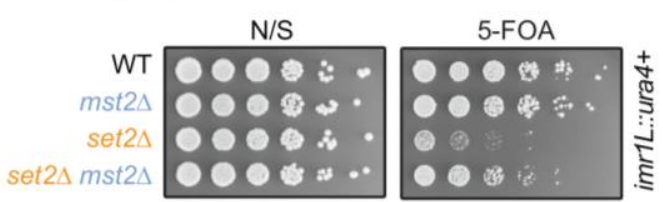

\section{subtelomeremes}

tel1L
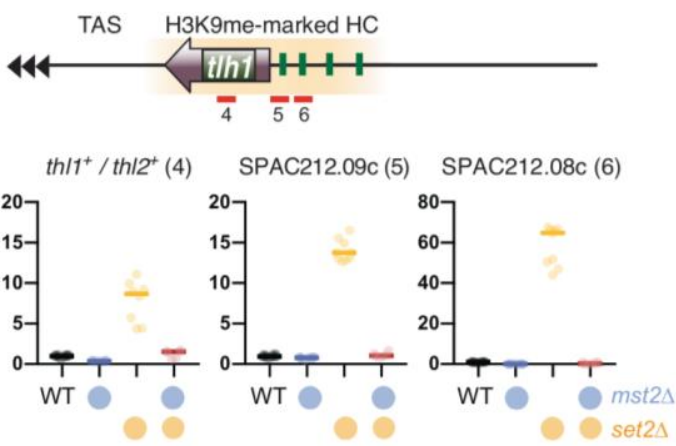

\section{subtelomeremes}

euchromatin
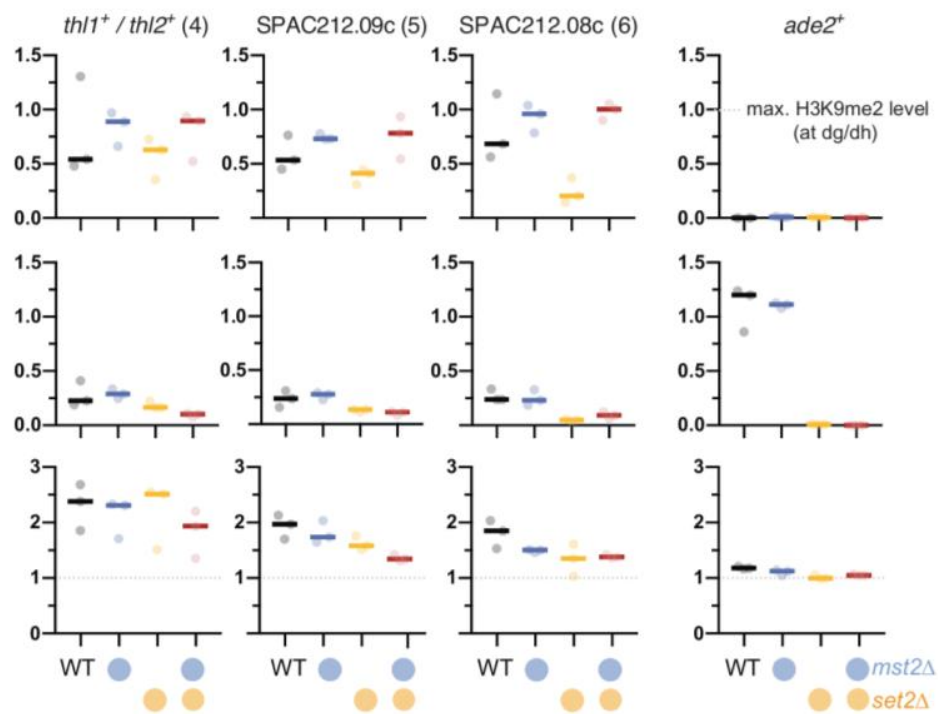

FIGURE 1: Loss of Mst2 recues the silencing defect caused by set2 ${ }^{+}$deletion. (A) Scheme depicting genetic interactions of set $2^{+}$, pdp $3^{+}$and $m s t 2^{+}$contributing to heterochromatic silencing and potential parallel pathways in which $\mathrm{H} 3 \mathrm{~K} 36 \mathrm{me} 3$ may be also involved. Black lines indicate positive regulations, red lines indicate negative regulations. (B) Silencing reporter assay with the imr::ura $4^{+}$reporter. Fivefold serial dilutions of wild-type (WT) cells and single and double deletion mutants of st2 $^{+}$and set2+; (N/S) nonselective medium. (C) RT-qPCR analysis. Shown are heterochromatic transcript levels of the strains used in (B). The schemes display the positions of the ura ${ }^{+}$reporter insertion and endogenous heterochromatic transcripts from pericentromeric (left) and subtelomeric heterochromatin (right); transcript levels have been normalized to $a c t 1^{+}$and are shown relative to WT for each transcript. Circles and horizontal lines represent individual data and median from 6-12 independent experiments. (D) ChIP-qPCR analysis for H3K9me2 (top), H3K36me3 (middle) and H3 (bottom) at pericentromeric and subtelomeric heterochromatin; ade ${ }^{+}$(right panels) was used as control for euchromatin. Circles and horizontal lines represent individual data and median from 3 independent experiments. Input-normalized ChIP data were corrected for variation in IP efficiency by normalizing to the mean of cen- $d g$ and cen-dh for $\mathrm{H} 3 \mathrm{~K} 9 \mathrm{me} 2$, or the mean of three euchromatic loci $\left(\right.$ tef $3^{+}$, ade $2^{+}$, act $1^{+}$) for $\mathrm{H}_{3 \mathrm{~K}} 36 \mathrm{me} 3$ and $\mathrm{H} 3$. $\mathrm{Note}$ that $\mathrm{H} 3 \mathrm{~K} 9 \mathrm{me} 2$ is largely unaltered at the $d h$ repeats in set $2 \Delta$ [34]. 
chromatic loci [34, 41-44] can be attributed to Mst2C mislocalization. This hypothesis makes the prediction that silencing will be restored when Mst2 is eliminated in set2 $\Delta$ cells, analogous to $m s t 2^{+}$deletion in a $p d p 3 \Delta$ strain (see scheme, Figure 1A). Silencing can be monitored in vivo using the $\mathrm{ura}^{+}$reporter gene inserted into a heterochromatic region. Presence of the nucleotide analog 5-FOA (5'fluoroorotic acid) inhibits cell growth due to the conversion of 5-FOA into a toxic metabolite by the gene product of ura $4^{+}$but allows growth when $u r a 4^{+}$transcription is repressed. By examining pericentromeric silencing in the imr1L::ura $4^{+}$reporter strain used previously [31], we found that growth of set $2 \Delta$ cells on $5-F O A$ is impaired, similar to pdp3 $\Delta$ cells and consistent with other studies that have reported silencing defects for set2 $\Delta[34,41-43]$. Remarkably, while cell growth in the presence of 5-FOA was not affected by lack of Mst2, it was nearly restored when $\mathrm{mst}^{+}$ was deleted in a set2 $\Delta$ background (Figure 1B). We confirmed the findings of the reporter assay by reverse transcriptase assays combined with quantitative PCR (RT-qPCR). Deletion of $\operatorname{set}^{+}$causes a reproducible upregulation of the imr1L::ura $4^{+}$reporter gene (4-fold) and two endogenous transcripts from the outer $d g$ and $d h$ repeats (both 3-fold; Figure 1C, left panels). In contrast, transcript levels in the set $2 \Delta$ mst2 $\Delta$ double mutant resemble those of wild-type (WT) cells. In addition, we examined expression levels of transcripts derived from a subtelomeric region that is marked with high levels of H3K9me2 (10-50 kb distal of the telomeric repeats, known as subtelomeric heterochromatin). De-repression of the subtelomeric genes $t / h 1^{+} / t / h 2^{+}$, SPAC212.09c and SPAC212.08c in set2 $\triangle$ cells is even more pronounced (10-, 15- and 60-fold, respectively; Figure 1C, right panels) than what we found at pericentromeres. Nonetheless, transcriptional upregulation at these loci is completely suppressed when $\mathrm{mst}^{+}$is concomitantly deleted in set $2 \Delta$ cells.

Constitutive heterochromatin in S. pombe is marked by high levels of H3K9me but largely devoid of euchromatic histone modifications (i.e. H3K4me, H3K36me) [43, 45]. By performing chromatin immunoprecipitation coupled to quantitative PCR (ChIP-qPCR), we observed a moderate H3K9me2 decrease in set2 $\Delta$ cells for several heterochromatic loci that display intermediate H3K9me2 levels (imr1L::ura4 ${ }^{+}$at pericentromeres; SPAC212.09c and $S P A C 212.08 \mathrm{c}$ at subtelomeres; Figure 1D, upper panels). Conversely, mst $2 \Delta$ and set $2 \Delta$ mst $2 \Delta$ cells showed elevated H3K9me2 levels at those loci, suggesting that Mst2 counteracts H3K9me2 in a chromatin context-dependent manner. This notion was further supported by a comprehensive analysis of $\mathrm{H} 3 \mathrm{~K} 9 \mathrm{me} 2$ using tiling oligonucleotides covering pericentromeric and subtelomeric heterochromatin, and a facultative heterochromatin island at the $\mathrm{mei}^{+}$locus (Figure S1). Especially at telomere-distal heterochromatin (30$50 \mathrm{~kb}$ downstream of telomeric repeats) and $\mathrm{mei}^{+}$, loss of Mst2 resulted in a large $\mathrm{H} 3 \mathrm{~K} 9 \mathrm{me} 2$ increase, suggesting that those chromatin regions are particularly prone to heterochromatin assembly.

While H3K4me is completely absent at constitutive heterochromatin [45], residual levels of H3K36me3 have been detected, particularly in the $S$ phase during which pericentromeric repeats are preferentially transcribed [34, 43]. We also found low H3K36me3 levels at pericentromeres and subtelomeres, reaching only $10-20 \%$ of the enrichment observed at euchromatin (Figure 1D, middle panels; note that the absolute level might even be lower, since the anti-H3K36me3 antibody used here shows limited cross-reactivity with H3K9me2; Figure S2). Importantly, H3K36me3 levels changed only marginally in set2 $\Delta$ and mst $2 \Delta$ single mutants at most heterochromatin loci examined and, as expected, remained low in cells lacking both Set2 and Mst2, despite of silencing being restored in the double mutant. We also analyzed nucleosome abundance by examining (total) histone H3. While H3 ChIP enrichments tend to be lower for some loci in these mutants, most changes were not significant and did not reflect transcriptional upregulation or changes in histone modifications (Figure 1D, lower panels). Together, these findings suggest that the silencing defects at pericentromeric and subtelomeric heterochromatin are not primarily caused by local changes of $\mathrm{H} 3 \mathrm{~K} 36 \mathrm{me} 3$ within heterochromatin (which is already low in WT cells). Rather, our results imply that they are triggered by the uncontrolled activity of Mst2, which in the absence of Set2 is no longer tethered to euchromatin and gains promiscuous genome-wide access to chromatin, including the heterochromatic regions [31].

\section{Set2 acts in the same genetic pathway as other Mst2C members}

We previously demonstrated that the Mst2C subunit Pdp3 mediates Mst2 recruitment via its PWWP domain that binds to H3K36me3 (Figure 2A, left panel) [31]. Since Set2 acts upstream of Pdp3 in Mst2 recruitment, we tested whether Set2 and Pdp3 also participate in the same pathway with respect to heterochromatin silencing. In agreement with our former findings [31], cells lacking Pdp3 displayed alleviated silencing at pericentromeres and subtelomeres, although we noted that the transcriptional increase was less pronounced in $p d p 3 \Delta$ than in set $2 \Delta$ (Figure 2B and C, left panels). Combining both deficiencies did not result in an additive increase; we rather observed a mild suppressive phenotype for $\operatorname{set} 2 \Delta p d p 3 \Delta$ when compared to the set $2 \Delta$ single mutant. Although the nature of the partial suppression remains unclear (see discussion), the nonadditive phenotype of the double mutant suggests that Pdp3 and Set2 act in the same pathway.

Besides Mst2 and Pdp3, Mst2C contains five additional subunits: Nto1, Eaf6, Tfg3, Ptf1 and Ptf2. Eaf6 is also present in the NuA4 acetyltransferase complex, while Tfg3 is shared with Ino80, SWI/SNF and the TFIID and TFIIF complexes. The functions of these subunits within Mst2C are not well understood, but Nto1 and Ptf2 are essential for the integrity and assembly of the complex, and mutants lacking either of these subunits phenocopy the loss of Mst2 [28]. We therefore tested whether deleting those subunits, analogous to $m s t 2^{+}$elimination, suppresses the silencing defect of $\operatorname{set} 2 \Delta$ (Figure 2A, right panel). Similar to $m s t 2 \Delta$, eliminating $n t o 1^{+}$, eaf $^{+}$and $p t f 2^{+}$alone did not increase gene repression but in combination with set $2 \Delta$ completely 
A

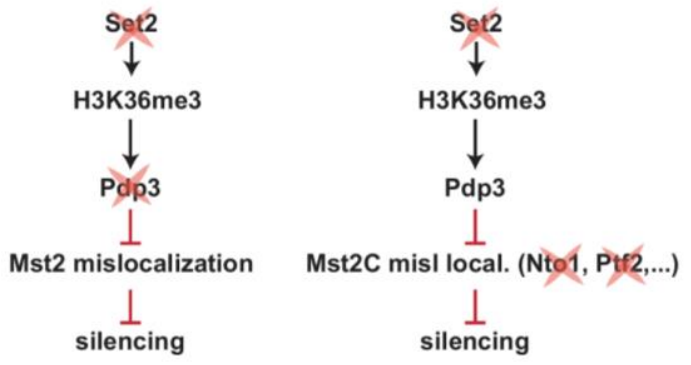

B RT-qPCR pericentromeres

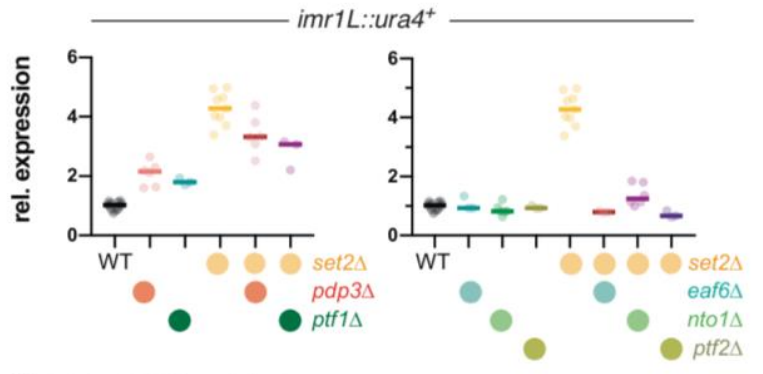

C RT-qPCR subtelomeremes
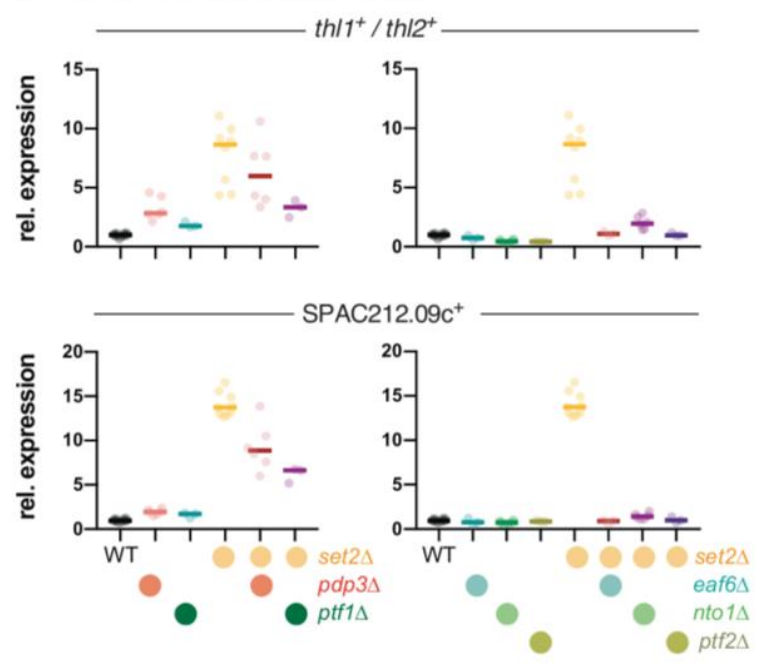

FIGURE 2: Loss of heterochromatin silencing in set2 $\Delta$ is dependent on functional Mst2C. (A) Scheme displaying genetic interactions and genes mutated for experiments shown in (B) and (C). (B, C) RT-qPCR analysis of transcript levels at pericentromeric (B) and subtelomeric HC (C) RT-qPCR data analysis and primer positions as in Figure 1C. Circles and horizontal lines represent individual data and median from 6 independent experiments unless specified (i.e. WT: $n=12$; ptf1 $\Delta$, ptf2 2 , eaf6 $\Delta$, ptf1 $\Delta$ set $2 \Delta$, ptf2 $\Delta$ set $2 \Delta$, and eaf $6 \Delta \operatorname{set} 2 \Delta: n=3$ ).

suppressed the silencing defect (Figure 2B and C, right panels). In contrast, deletion of $p t f 1^{+}$rather resembled the phenotype of $p d p 3 \Delta$, showing a moderate upregulation and partial suppression in combination with $\operatorname{set} 2 \Delta$, suggesting that Ptf1 also contributes to the recruitment of Mst2C to H3K36me3 (Figure 2B and C, left panels). We refrained from examining the $t f g 3^{+}$, as its presence in multiple complexes likely causes pleiotropic effects that may be difficult to interpret. From these data we conclude that an intact Mst2 complex is required to trigger the silencing defect at heterochromatin.

Mst2-dependent silencing defects are not mediated through Brl1 acetylation

We previously showed that Mst2 is involved in the acetylation of the non-histone substrate Brl1, a conserved ubiquitin $\mathrm{E} 3$ ligase that mono-ubiquitylates histone $\mathrm{H} 2 \mathrm{~B}$ at lysine 119 [31]. Acetylation of Brl1 at lysine 242 (Brl1-K242ac) may have a stimulatory effect on its enzymatic activity (H2B-K119ub) and downstream events (H3K4me3), which protects euchromatic genes against the ectopic formation of heterochromatin, likely through increased transcription [31]. We therefore wondered whether the Brl1-K242acdependent positive feedback loop is the main cause for the silencing defect observed in set $2 \Delta$ cells (Figure $3 A$ ). By ChIP, we confirmed previous reports $[46,47]$ that H2B-K119ub is low at heterochromatic regions but highly enriched over the gene body of actively transcribed genes (Figure 3B). Interestingly, H2B-K119ub was significantly reduced in the absence of Set2 at several of euchromatic genes but not at act $^{+}$(Figure S3), which we used as an internal control for normalization in the subsequent ChIP experiments. We found that cells expressing the br/1-K242R mutant that inhibits Brl1 acetylation also showed decreased H2BK119ub at several euchromatic genes $\left(\right.$ ade $^{+}, \mathrm{mst2}^{+}$, pyk1 ${ }^{+}$ but not tef $3^{+}$, Figure $3 \mathrm{C}$ ) yet less distinct than seen in set $2 \Delta$ cells. The set $2 \Delta$ br/1-K242R double mutant displayed a slightly enhanced decrease, whereas the brl1-K242Q mimicking Brl1 acetylation did neither alter H2B-K119ub in WT nor in set $2 \Delta$ cells. Together, these data imply that mutations affecting Brl1 acetylation regulate $\mathrm{H} 2 \mathrm{~B}$ ubiquitylation in euchromatin, which is in agreement with our previous observation for total H2B-K119ub [31] (although it appears that Set2 also contributes through a Mst2-independent pathway).

However, when examining heterochromatin, we found hardly any change in these mutants for H2B-K119ub, which was 5-10 times lower than euchromatin in WT cells and remained low in $b r l 1-K 242 R$ and set2 $\Delta$ cells (Figure 3D). Moreover, in stark contrast to set $2 \Delta$ mst2 $2 \Delta$ cells (Figure 1C), we found that silencing at pericentromeres and subtelomeres was not reinstated in the set2 $\triangle$ brl1-K242R double mutant (Figure 3E). Hence, preventing Brl1 acetylation is not sufficient to block the anti-silencing activity of Mst2. Instead, we observed transcriptional upregulation even in the single brl1-K242R mutant for some heterochromatic loci, suggesting that the loss of its acetylation target Brl1 (in euchromatin) renders Mst2 more active (in heterochromatin). From this we conclude that Mst2's euchromatin-protective role via Brl1-K242ac differs from its role in counteracting gene silencing at heterochromatin. This further implies that Mst2 targets at least one other substrate that is critical for heterochromatin silencing. 
A

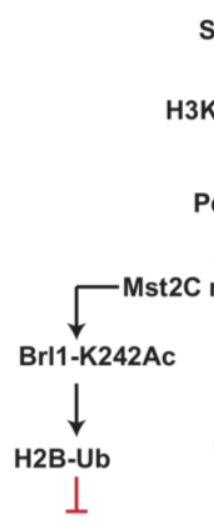

silencing
Set2

$\downarrow$

H3K36me3
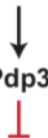

mislocal.

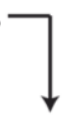

?

$\mathrm{X}$

silencing
B ChIP H2B-K119ub (WT)

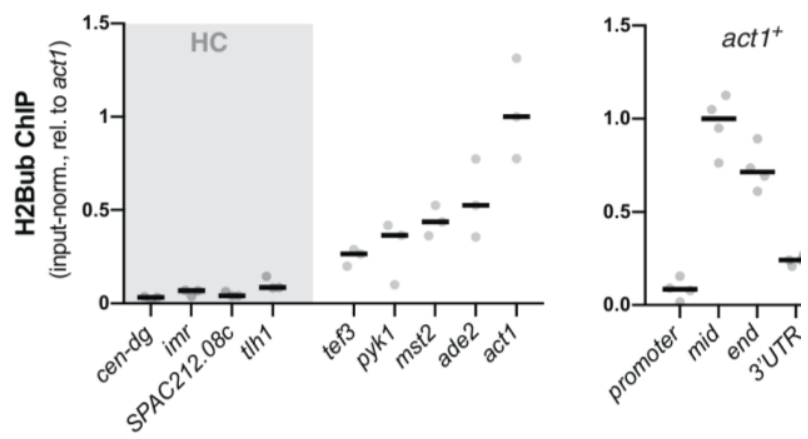

\section{ChIP H2B-K119ub euchromatin}
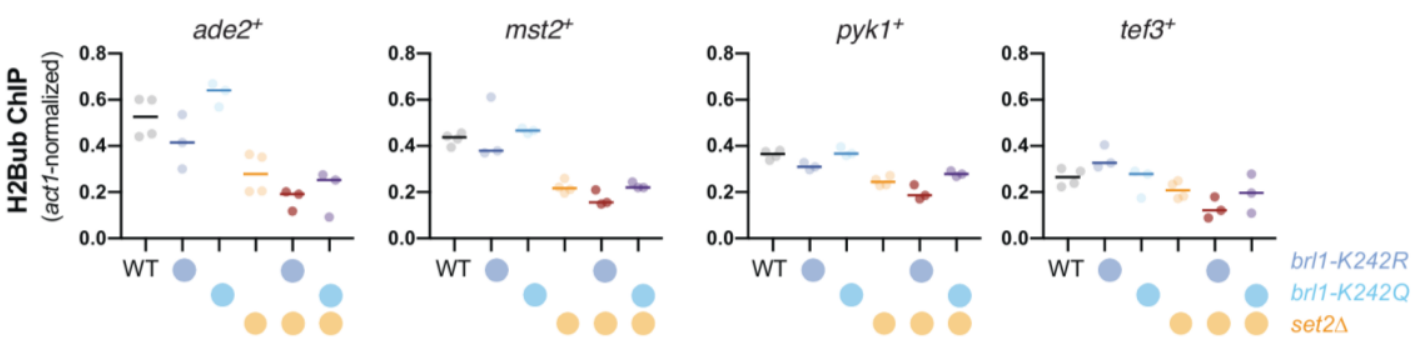

D ChIP H2B-K119ub

\section{heterochromatin}
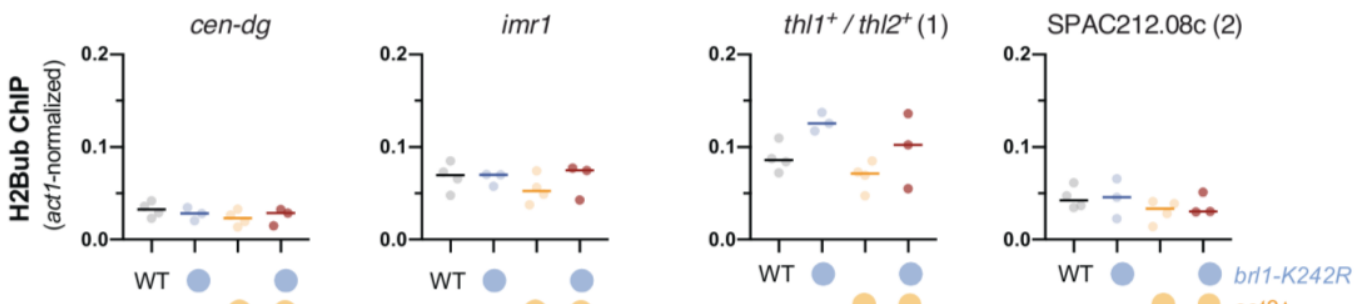

E RT-qPCR pericentromeres
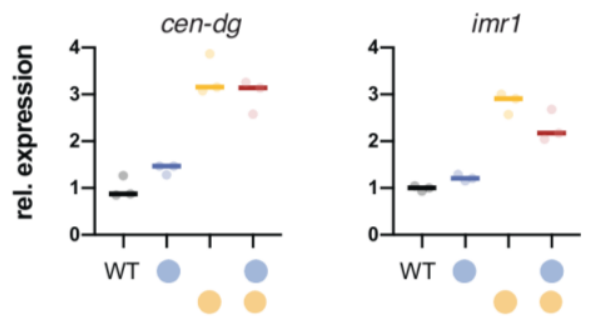

\section{subtelomeres}
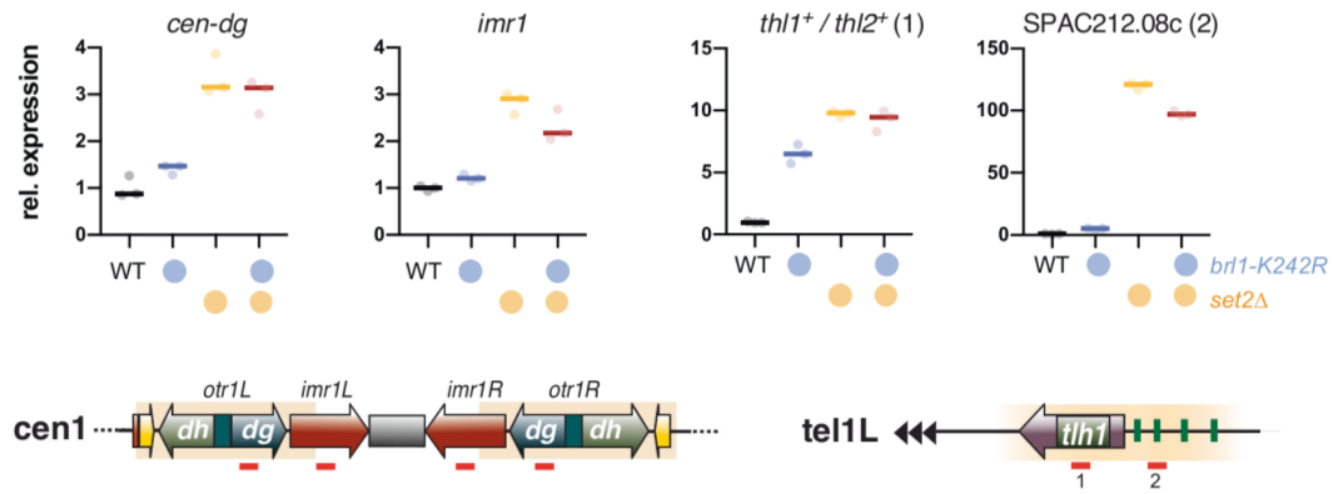

FIGURE 3: The target of Mst2 in heterochromatin is not Brl1. (A) Scheme depicting the described Mst2C pathway [31] involving Brl1-K242 acetylation and $\mathrm{H} 2 \mathrm{~B}$ ubiquitylation and a potential alternative pathway on $\mathrm{HC}$ silencing; black arrows represent positive regulation and red lines represent negative regulation. (B) ChIP-qPCR analysis for H2B-K119ub in wild-type cells. Circles and horizontal lines represent individual data and median from 3 (left panel) and 4 independent experiments (right panel). Input-normalized ChIP data are shown relative to the median of the ChIP signals for act $1^{+}$(mid). (C, D) ChIP-qPCR analysis for H2B-K119ub in mutants affecting Brl1 acetylation. Circles and horizontal lines represent individual data and median from 3 independent experiments (except: WT, set2 $\Delta: n=4)$. ChIP analysis as in (B), except that ChIP data were corrected for variation in IP efficiency by normalizing to $a c t 1^{+}$(mid). Note that H2B-K119ub at act $1^{+}$is not largely affected by Set2. (E) RT-qPCR analysis of transcript levels at pericentromeric and subtelomeric HC. Data analysis as in Figure $1 \mathrm{C}$ ( $n=3$ individual experiments). 
Silencing defects in set2 $\Delta$ at other repressed loci involve an Mst2-independent pathway

Loss of set2 $2^{+}$does not only affect gene repression at chromatin regions with high levels of H3K9me2 but also other subtelomeric loci. These include the telomere-proximal region (300 bp - $10 \mathrm{~kb}$, known as telomere-associated sequences, TAS), as well as a region $50 \mathrm{~kb}$ downstream of the telomeric repeats, which is characterized by highly condensed chromatin bodies dubbed 'knobs' [44] (Figure 4A and $\mathbf{B}$ ). Transcription of the non-coding RNA TERRA (telomeric repeat-containing non-coding RNA) from the TAS is repressed by heterochromatin and members of shelterin, the telomere-end protecting complex [48, 49]. However, this subtelomeric region displays low nucleosome abundance [50] and establishes only low levels of H3K9me2 and H3K36me3 (Figure 4C and D). Similarly, subtelomeric 'knob' genes are decorated with a low amount of H3K9me2 and display reduced H3K36me3 compared to euchromatin (Figure 4C and D).

When we examined gene expression at the TAS, we found a subtle but reproducible upregulation (2 to 3-fold) of TERRA in set2 $\triangle$. A similar increase was also observed in $m s t 2 \Delta$, and concomitant deletion of $\mathrm{mst}^{+}$in the set2 $\Delta$ mutant did not suppress the silencing defect (Figure 4E, left panel). In agreement with previous studies, we also detected a 2 to 10 -fold upregulation for several 'knob' genes [34, 44]. Additional deletion of $\mathrm{mst}^{+}$did not restore silencing of these loci in $\operatorname{set} 2 \Delta$ (or caused only a partial suppression; Figure $4 \mathrm{E}$ ). Nonetheless, disruption of $\mathrm{mst}^{+}$ promoted the establishment of H3K9me2 at several knob genes (particularly SPAC977.15), corroborating the notion of Mst2C playing a global role in antagonizing heterochromatin $[30,31,51]$. However, since removal of Mst2 was not sufficient to reinstate silencing in the absence of Set2, we presume that an additional, Mst2- and H3K9meindependent pathway interferes with the repression of these genes.

\section{DISCUSSION}

Set2-dependent silencing defects in heterochromatin are functionally linked to Mst2C

Transcriptionally active and repressed chromatin regions are marked by different posttranslational modifications. H3K36me3 in particular is deposited co-transcriptionally through the recruitment of Set2 by transcribing RNAPII and likely other elongating factors [35]. Yet paradoxically, loss of Set2 also causes defects in transcriptionally repressed heterochromatin [34, 41-43]. We previously have shown that deleting mst2 restores silencing in cells lacking the H3K36me3 reader Pdp3 [31]. Here, we demonstrate that eliminating the catalytic KAT subunit Mst2, or other subunits critical for Mst2C assembly, fully reverses the silencing defect of Set2-deficient cells at constitutive heterochromatin regions (Figure $\mathbf{1}$ and $\mathbf{2}$ ). Reinstatement of silencing is not only seen at the level of transcription but also at the level of heterochromatin structure (H3K9me2) at loci where loss of Set2 affects both (Figure 1). In contrast, $\mathrm{H} 3 \mathrm{~K} 36 \mathrm{me} 3$ is low at these heterochromatin regions even in WT cells and also not affected upon deletion of $m s t 2^{+}$. This suggests that defective silencing is not caused directly by the loss of $\mathrm{H} 3 \mathrm{~K} 36 \mathrm{me}$ within heterochromatin but rather indirectly through the promiscuous activity of Mst2C. Although Set2 is implicated in various cellular functions, pleiotropic defects appear to be less likely the cause of impaired silencing. Instead, the fact that silencing is completely restored at constitutive heterochromatin implies that Set2 exclusively controls gene repression through sequestration of Mst2 by H3K36me3. It is worth mentioning that although Mst2 is not detected by ChIP at transcribed chromatin in $p d p 3 \Delta$ cells, it has still access to chromatin, as we previously demonstrated by DamID experiments [31].

Recruitment of Mst2 to euchromatin is mediated by the Mst2C subunit Pdp3, which binds to H3K36me3 via its PWWP domain. Consistently, lack of Pdp3, or a point mutation within its PWWP domain, also produces a defect in heterochromatin silencing [31]. However, we noticed that the silencing defect in $p d p 3 \Delta$ is less pronounced than in set2 $\Delta$ (Figure 2), despite the fact that silencing can be fully restored in the absence of Mst2 (see Figure 1). A possible explanation would be that Mst2 recruitment involves another H3K36me3-binding factor that acts redundantly with Pdp3. Indeed, the Mst2C subunit Nto1 contains two PHD (plant homeodomain) fingers, and the $S$. cerevisiae homolog shows affinity for H3K36me3 [52]. However, since Nto1 is essential for Mst2C assembly, a putative role in restricting Mst2 to euchromatin would be masked by the complete loss of KAT activity, resulting in deviating phenotypes for $p d p 3 \Delta$ and $n t o 1 \Delta$ mutants. Alternatively, Pdp3 may also contribute to the stability or activity of the complex (at least in part), in addition to its function in $\mathrm{H} 3 \mathrm{~K} 36 \mathrm{me} 3$ anchoring. This could explain the intermediate phenotype of pdp $3 \Delta$ cells compared to set $2 \Delta$ on one hand, and mst $2 \Delta$ on the other. More work will be needed to better understand the functions of the individual subunits of Mst2C.

\section{Mst2C has distinct cellular functions by acetylating multi- ple targets}

Mst2 acetylates H3K14 in vitro and in vivo and acts redundantly with the SAGA member Gcn5 [28]. H3K14ac is critical for G2/M checkpoint activation upon DNA damage and controls chromatin compaction through recruitment of the nucleosome remodeler RSC [28]. H3K14ac also accumulates in heterochromatin upon deletion of the HDAC Clr3 and other components of the repressor complex SHREC, suggesting a function in antagonizing heterochromatin silencing $[10,53]$. Moreover, the anti-silencing factor Epe1 physically interacts with SAGA and targets the HAT to heterochromatin when Epe1 is overexpressed. This triggers a silencing defect that is accompanied by an H3K14ac increase and is dependent on the HAT activity of Gon5 [54]. At first glance, this seems reminiscent of the phenotype caused by relocalization of Mst2. However, additional findings cast doubt on whether H3K14 is the relevant substrate that mediates the anti-silencing function of Mst2. First, neither lack of Set2 nor of Pdp3 (both causing delocalization of Mst2) results in H3K14ac accumulation at heterochromatin, as we and other showed previously [31, 34] Second, while elimination of Mst2 bypasses the need for 

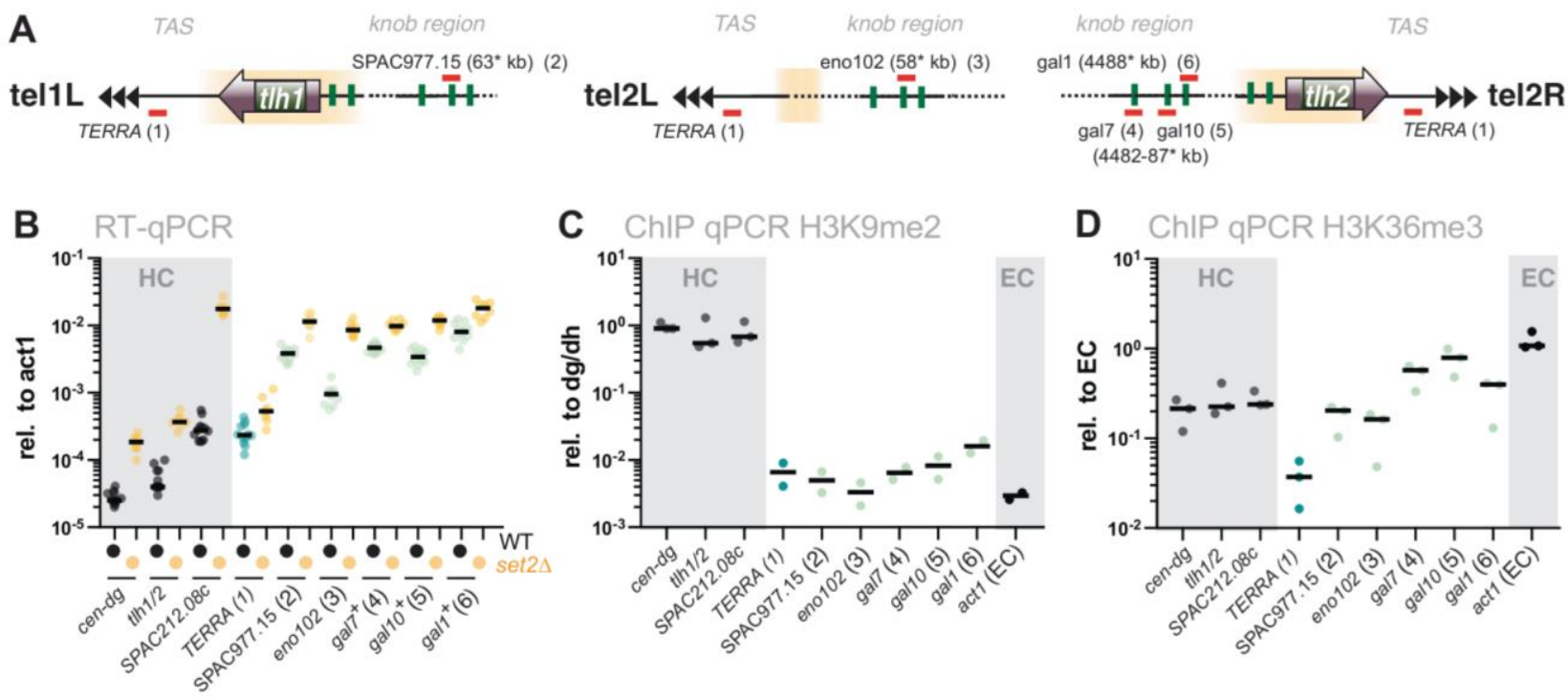

C ChIP qPCR H3K9me2

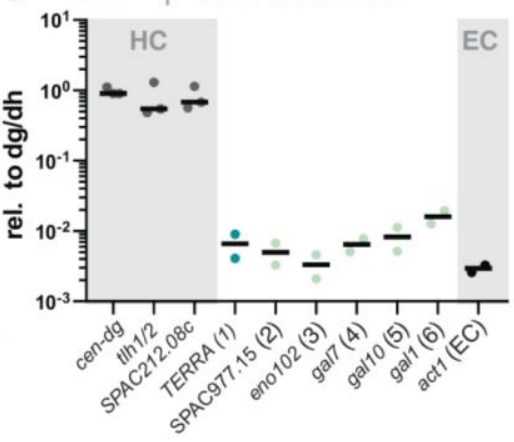

\section{ChIP qPCR H3K36me3}

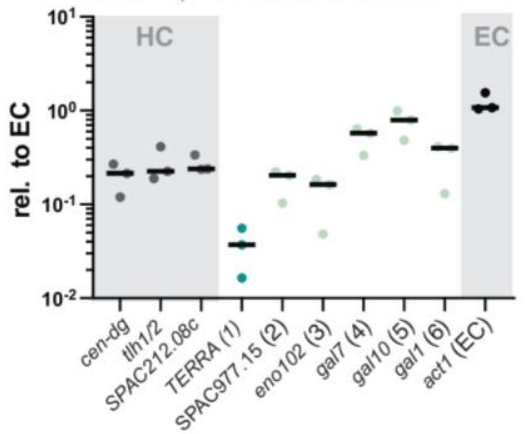

\section{E $\quad$ RT-qPCR}
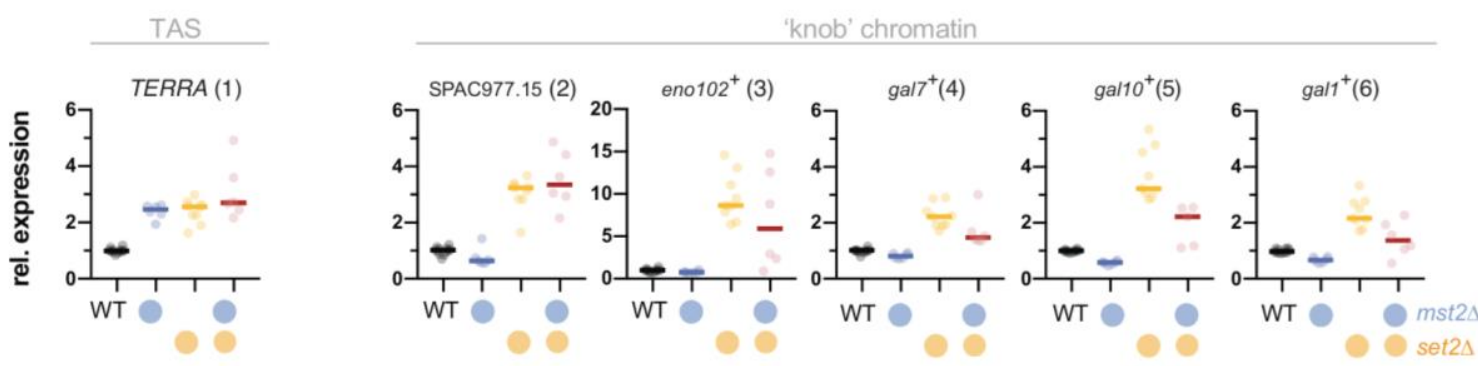

$\mathbf{F}$

ChIP-qPCR (H3K9me2, H3K36me3, H3)

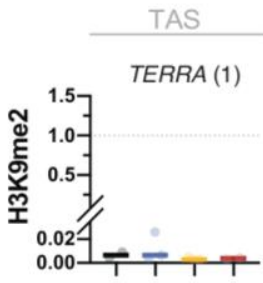

'knob' chromatin
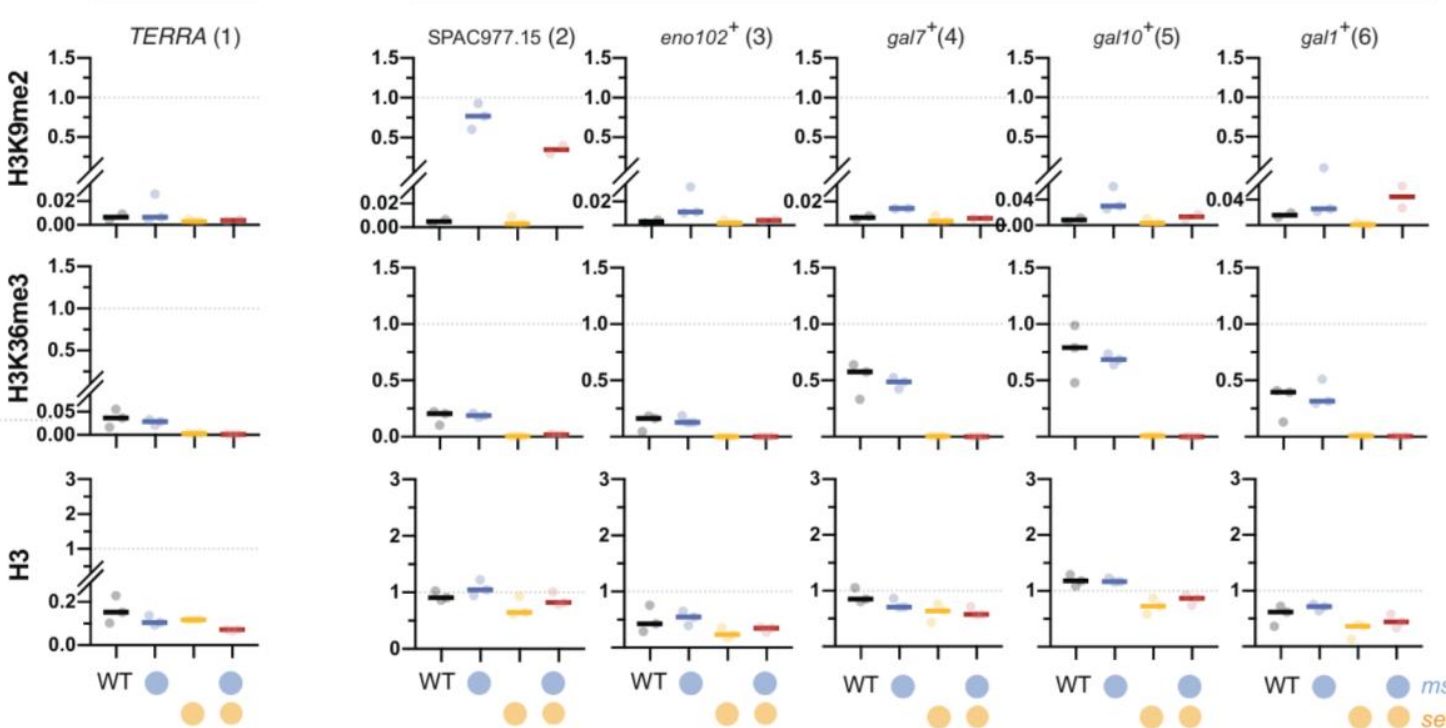

FIGURE 4: The silencing defect at 'knobs' in set2 $\Delta$ is not associated with the Mst2 pathway. (A) Scheme depicting expression sites of the non-coding RNA TERRA and the positions of several loci within the 'knob' regions on chromosomes I and II. Chromosomal positions refer to annotations in www.pombase.org but differ from the absolute positions due to missing sequences at the chromosomal termini. (B) RT-qPCR analysis in WT and set2 $\Delta$ strains comparing transcript levels at pericentromeric and subtelomeric HC (shaded in grey) to loci in the 'knob' region. Transcript levels from 12 independent experiments are shown relative to act1 ${ }^{+}$. (C, D) ChIP-qPCR analysis of H3K9me2 and H3K36me enrichments in WT cells at loci analyzed in (B). Shown are ChIP data from 2-3 individual experiments analyzed as described in Figure 1D. (E) RT-qPCR analysis in $m s t 2 \Delta$ and set $2 \Delta$ single and double mutants; data from independent experiments analyzed as described in Figure $1 C$ (WT $n=12 ;$ mst2 2 , mst2 $\operatorname{set} 2 \Delta: n=6$; set2 $\Delta: n=9$ ). (F) ChIP-qPCR analysis of TERRA and different loci located within the 'knob' region; data from independent experiments analyzed as described in Figure 1D $(n=3)$. 
RNAi in pericentromeric silencing, this is not the case for mutants lacking Gcn5 [30]. Third, lack of Mst2 but not Gcn5 promotes the assembly of ectopic heterochromatin domains [31]. Together, these findings suggest a nonredundant function of Mst2 in antagonizing heterochromatin that likely involves another substrate than H3K14.

We previously showed that the euchromatin-protective role of Mst2 is mediated through acetylation of the nonhistone substrate Brl1, a subunit of HULC [31]. In particular, replacing $b r / 1^{+}$with an acetylation-deficient mutant (brl1$K 242 R$ ) phenocopied the deletion of $\mathrm{mst}^{+}$, whereas mimicking acetylation (br/1-K242Q) bypassed the need for Mst2 in protecting euchromatin. In agreement with this finding, deletion mutants of $\mathrm{brl}^{+}$or other components of HULC display more robust heterochromatin silencing than WT cells [47]. Thus, acetylated Brl1 seemed to be a likely candidate for mediating the anti-silencing function of Mst2 also at heterochromatin. However, in contrast to mst2 $\Delta$, we found that introducing the $b r / 1-K 424 R$ mutant was not sufficient to suppress the silencing defect of $\operatorname{set} 2 \Delta$. While initially we did not expect a Brl1-independent Mst2 function at heterochromatin, several lines of evidence support the idea that Mst2 acts through different pathways at euand heterochromatin. First, whereas Mst2 antagonizes heterochromatin even when not recruited by H3K36me3, $\mathrm{Brl} 1$ acetylation and euchromatin protection requires stable chromatin binding of Mst2 via Pdp3 [31]. Consistently, we found that H2B-K119ub levels are altered in set2 and brl1 mutants at several euchromatic genes but not at heterochromatin (Figure $\mathbf{3 C}$ and $\mathbf{D}$ ). Second, at heterochromatin, loss of Pdp3 or Set2 causes de-repression and H3K9me decrease, whereas lack of Mst2 results in enhanced repression and increased H3K9me. However, at euchromatin and facultative heterochromatin, the lack of either Pdp3 or Mst2 causes ectopic heterochromatin assembly (Figure S1; [31]). Finally, for several subtelomeric genes, we observed a synthetic defect in gene repression when combining set $2 \Delta$ with the acetylation-mimicking brl1-K242Q mutant, suggesting that both pathways can affect these loci (Figure S4). We therefore speculate that besides H3K14 and Brl1K242 Mst2C targets at least one other substrate that is important for heterochromatin maintenance (Figure 5). Previous proteomics failed so far to identify additional substrates besides Brl1 that involve acetylation by Mst2 [31]. However, since heterochromatin makes up only a small portion of the genome and Mst2 may have only transient access to these genomic regions, a heterochromatinspecific substrate would be more difficult to identify.

\section{Set2-mediated gene repression at chromatin lacking H3K9me is independent of Mst2C}

Besides defects at constitutive heterochromatin, deletion of $\operatorname{set}^{+}$results in the transcriptional upregulation of other genes that are part of repressed chromatin regions but largely devoid of $\mathrm{H} 3 \mathrm{~K} 9 \mathrm{me} 2$. These include the non-coding RNA TERRA expressed from the telomere-proximal TAS region as well as various other genes expressed from the telomere-distal 'knob' region, which is characterized by high chromatin condensation and the absence of most post-transcriptional histone modifications [44]. However, except for TERRA, we still detect some H3K36me3 at these chromatin regions, albeit reduced compared to euchromatin (Figure 4). Transcriptional upregulation of these loci is moderate in set2 $\Delta$ and not suppressed by concomitant Mst2 elimination. Nonetheless, Mst2 may still gain access to these loci and antagonize heterochromatin formation, as seen by increased levels of H3K9me2 upon mst $^{+}$deletion. Nonetheless, the lack of suppression suggests the involvement of a Set2-dependent but Mst2-independent pathway that is critical for gene repression.

Another histone-modifying complex potentially involved in Set2-dependent silencing is the HDAC CIr6C-II, which contains the chromodomain protein Alp13 (Eaf3 in S. cerevisiae; MORF4 in humans) $[41,55]$. The homologous complex in S. cerevisiae, Rpd3S, prevents cryptic transcription through deacetylation of histones in coding regions marked with H3K36me2/3 via Eaf3 [36-39]. Similarly, Clr6CII promotes deacetylation of $\mathrm{H} 3 \mathrm{~K} 9$ in coding regions, and both Alp13 and Set2 prevent antisense transcription and repress pericentromeric repeats [41]. In addition, both H3K36me3 and Alp13 accumulate on heterochromatin during $S$ phase when pericentromeric repeats are transcribed [43]. However, in contrast to its S. cerevisiae homo$\log$, S. pombe Set2 does not contribute to deacetylation of bulk histones and only moderately affects antisense transcription; in addition, the set $2 \Delta$ alp $13 \Delta$ double mutant displays an additive defect, thus arguing for parallel pathways [41]. Moreover, while H3K36me2 is sufficient to recruit budding yeast Rpd3S [56], heterochromatin silencing and recruitment of $\mathrm{Pdp} 3 / \mathrm{Mst} 2 \mathrm{C}$ requires H3K36me3 [31, 34].

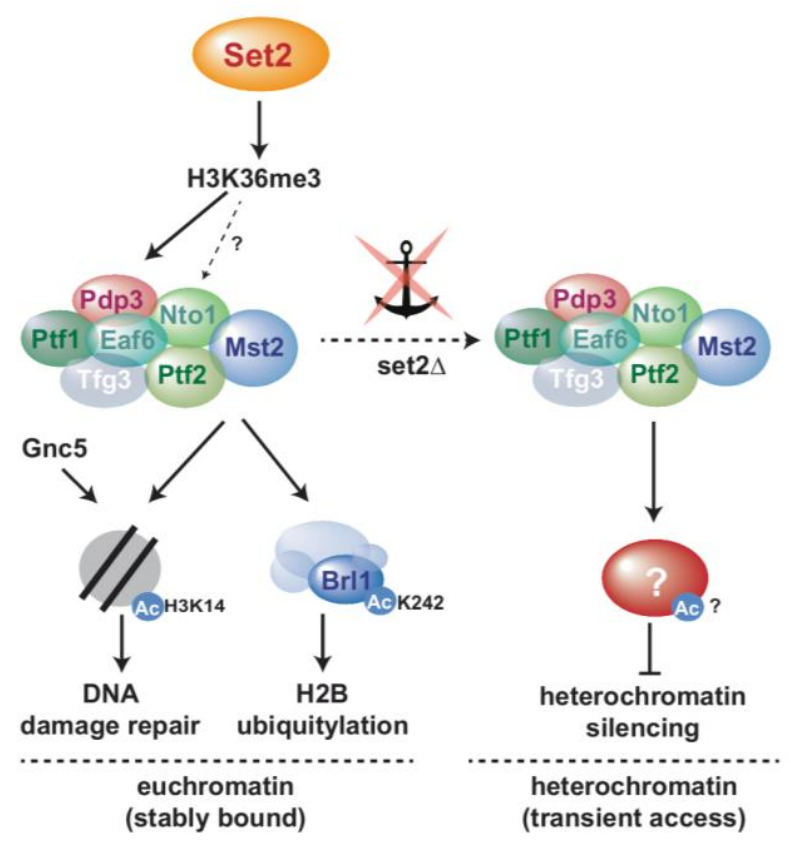

FIGURE 5: Model for Mst2C-dependent functional pathways in the presence of $\mathrm{H} 3 \mathrm{~K} 36 \mathrm{me} 3$ (sequestered to euchromatin) and in the absence of H3K36me3-mediated anchoring (promiscuous access to heterochromatin). 
Together with the fact that silencing is fully restored in the absence of Mst2, it therefore appears less likely that Clr6CII contributes significantly to the Set2-dependent pathway at heterochromatin. Still, it remains an attractive hypothesis that Clr6C-II represses transcription in an H3K36me/Alp13-dependent manner at other loci where Mst2 plays a less prevailing role.

\section{H3K36me-mediated KAT sequestration is conserved in heterochromatin maintenance}

In worm embryos, perinuclear heterochromatin is established through two methyltransferases (MET-2, SET-25) and the nuclear membrane-associated chromodomain protein CEC-4, which tethers H3K9me-marked chromatin to the nuclear periphery $[57,58]$. However, during differentiation, this pathway becomes redundant. An RNAi screen in cec-4-null larvae identified the chromodomain protein MRG-1 as a critical factor for perinuclear heterochromatin organization [59]. MRG-1 is homologous to the Rpd3S subunit Eaf3 (S. cerevisiae) and Clr6C-II subunit Alp13 (S. pombe). In addition, MRG-1 may associate with the HAT CBP-1/p300 analogous to other organisms. Although MRG-1/CBP-1 and Pdp3/Mst2C are not homologous to each other, there are still striking parallels [59]: Like Pdp3, MRG-1 binds to H3K36me3-marked euchromatin. Loss of perinuclear heterochromatin in $\mathrm{mrg}$-1-null is phenocopied by the double mutant lacking the H3K36 methyltransferases MET-1 and MES-4. Reducing CBP-1 restores silencing in $\mathrm{mrg}$-1-null larvae, whereas overexpression of $c b p-1$ is sufficient to release heterochromatin from the nuclear periphery in the absence of CEC-4. Moreover, the authors detected increased CBP-1 binding at several heterochromatic genes in mrg-1-null larvae. This was accompanied by elevated histone acetylation (H3K27ac), providing a direct link to gene expression.

Altogether, this demonstrates that the principle of heterochromatin maintenance through internal sequestration of KATs is conserved between fission yeast and worms, despite some apparent differences regarding the molecular mechanisms (i.e. the nature of the KAT enzymes and their substrates). It further unveils that the pathways partition in eu- and heterochromatin are remarkably entwined, requiring spatial constraint of opposing chromatin activities to maintain the identity of chromatin states. Recent observations have reinforced the notion that repressive histone marks contribute to epigenetic inheritance of chromatin domains $[15,16,27,60]$. In contrast, histone modifications associated with euchromatin have been considered rather a consequence than a cause of transcription. The discovery of H3K36me3 as a critical factor in heterochromatin maintenance will likely reopen the discussion to what extent 'active' marks also contribute to the epigenetic states of chromatin.

\section{MATERIALS AND METHODS}

\section{Contact for reagent and resource sharing}

Important reagents and assays used are listed in Table S5. Further information and requests for resources and reagents should be directed to and will be fulfilled by Sigurd Braun (sigurd.braun@bmc.med.Imu.de).

\section{Yeast techniques and strains}

Standard media and genome engineering methods were used [61]. For the $\mathrm{ura}^{+}$reporter assay in Figure $\mathbf{1 B}$ cells were plated on EMM or EMM containing $1 \mathrm{mg} / \mathrm{mL} F O A$. The strains were grown at $30^{\circ} \mathrm{C}$ for three (non-selective, NS) and four days (5-FOA), respectively. Cultures were grown at $30^{\circ} \mathrm{C}$ in liquid YES media (160 rpm, 12-24 hours) or at $30^{\circ} \mathrm{C}$ on solid YES agarose plates (for 3 days). The brl1-K242R point mutant was provided by M. Bühler (FMI, Basel). Strains used in this study are listed in Table S1.

\section{RT-qPCR analyses}

RT-qPCR experiments were carried out as previously described [62]. The data are presented as individual data points together with the median. CDNA was quantified by qPCR using the primaQUANT CYBR Master Mix (Steinbrenner Laborsysteme) and a QuantStudio 5 Real-Time PCR System (Applied Biosystems) and primers listed in Table S2. Prior to calculation of the median, $a c t 1^{+}$normalized data sets from independent experiments were standardized to the mean of all samples from each experiment (experimental normalization; eq 1.1). These sample pool-normalized results were shown relative to the mean value of the sample pool-normalized WT data from all (n) experiments (eq 1.2).

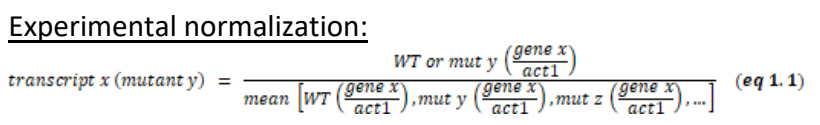

Mean WT normalization:

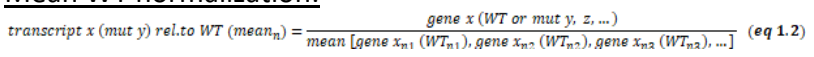

Using the average from a collection (sample pool) instead of a single strain (e.g. WT) reduces bias, especially when transcripts levels are low in the repressed state and therefore more prone to noise.

\section{ChIP assays}

ChIP experiments were performed essentially as described [21]. Cross-linking was performed with $1 \%$ formaldehyde for $10 \mathrm{~min}$ at room temperature. For quantitative ChIP, immunoprecipitations were performed with $2 \mu \mathrm{g}$ of the following antibodies (cell lysates corresponding to different amounts of $\left.\mathrm{OD}_{600}\right)$ : anti-H3K9me2 (15 OD $\left.\mathrm{O}_{600}\right)$ anti-H3K36me3 (5 OD $\left.\mathrm{OD}_{600}\right)$ anti-H3 $\left(5 \mathrm{OD}_{600}\right)$ and anti-H2B-K12Oub $\left(20 \mathrm{OD}_{600}\right)$. Antibodies are listed in Table S4. Immunoprecipitated DNA was quantified by qPCR using the primaQUANT CYBR Master Mix (Steinbrenner Laborsysteme) and a QuantStudio 5 Real-Time PCR System (Applied Biosystems). Primers are listed in Table S2. Unless otherwise noted, the median was calculated from three independent experiments. qPCR signals were normalized against the input samples for each primer position as internal control. For ChIP experiments with anti-H3K9me2, the inputnormalized values were corrected for variation by normalizing against the mean of cen-dg and cen-dh as the otr is the region with the highest and most stable H3K9me2 enrichment (' $\mathrm{HC}$ normalized', eq 2.1). For ChIP experiments with anti$\mathrm{H} 3 \mathrm{~K} 36 \mathrm{me} 3$ and $\mathrm{H} 3$, input-normalized qPCR signals were normalized to the mean of 3 euchromatic loci $\left(a c t 1^{+}\right.$, ade $2^{+}$, tef $^{+}$) 
as an internal control, which was set to 1 ('EC normalized', eq 2.2). ChIP results with anti-H2B-K119ub were analyzed analogously, except that ChIP signals were normalized to input (Figure 3B and Figure S3) or $a c t 1^{+}$as an internal control (Figure 3C and $\mathbf{D})$.

\section{Internal ChIP normalization H3K9me2:}

rel. $C h I P($ HC norm. $)=\frac{\text { locus } x\left(\frac{\text { ChIP }}{\text { input }}\right)}{\operatorname{mean}\left[\operatorname{cen}-d g\left(\frac{C h I P}{\text { input }}\right) \cdot \operatorname{cen}-d h\left(\frac{C h I P}{\text { input }}\right)\right]}$

Internal ChIP normalization H3K6me3 and H3:

rel.ChIP $(E C$ norm. $)=\frac{\text { locus } x\left(\frac{\text { ChIP }}{\text { input }}\right)}{\operatorname{median}\left[\operatorname{act} 1\left(\frac{C h I P}{\text { input }}\right), \text { ade2 }\left(\frac{\text { ChIP }}{\text { input }}\right), \text { tef3 } 3\left(\frac{C h I P}{\text { input }}\right)\right]} \quad($ eq 2.2)

Using the mean of multiple euchromatic loci ('EC') instead of single locus (e.g. $a c t 1^{+}$) reduces bias coming from variations in ChIP experiments, especially when doing IP experiments with bulk histones.

\section{ACKNOWLEDGMENTS}

We thank Marc Bühler and Michaela Smolle for critical reading of the manuscript. We further thank Marc Bühler for the brl1 point mutant fission yeast strains. This work was supported by grants awarded to SB from the German Research Foundation (BR 3511/2-1, BR 3511/4-1). SB is a member of the Collaborative Research Center 1064 funded by the German Research Foundation and acknowledges infrastructural support.

\section{REFERENCES}

1. Cavalli G, and Heard E (2019). Advances in epigenetics link genetics to the environment and disease. Nature 571(7766): 489-499. doi: 10.1038/s41586-019-1411-0

2. Allshire RC, and Madhani HD (2018). Ten principles of heterochromatin formation and function. Nat Rev Mol Cell Biol 19(4): 229-244. doi: 10.1038/nrm.2017.119

3. Grewal SIS, and Jia S (2007). Heterochromatin revisited. Nat Rev Genet 8(1): 35-46. doi: 10.1038/nrg2008

4. Nakayama J, Rice JC, Strahl BD, Allis CD, and Grewal SI (2001). Role of histone $\mathrm{H} 3$ lysine 9 methylation in epigenetic control of heterochromatin assembly. Science 292(5514): 110-113. doi: 10.1126/science. 1060118

5. Allshire RC, and Ekwall K (2015). Epigenetic Regulation of Chromatin States in Schizosaccharomyces pombe. Cold Spring Harb Perspect Biol 7(7): a018770. doi: 10.1101/cshperspect.a018770

6. Iglesias N, Currie MA, Jih G, Paulo JA, Siuti N, Kalocsay M, Gygi SP, and Moazed D (2018). Automethylation-induced conformational switch in Clr4 (Suv39h) maintains epigenetic stability. Nature 560(7719): 504-508. doi: 10.1038/s41586-018-0398-2

7. Zhang K, Mosch K, Fischle W, and Grewal SIS (2008). Roles of the Clr4 methyltransferase complex in nucleation, spreading and maintenance of heterochromatin. Nat Struct Mol Biol 15(4): 381-388. doi: 10.1038/nsmb.1406

8. Motamedi MR, Hong E-JE, Li X, Gerber S, Denison C, Gygi S, and Moazed D (2008). HP1 proteins form distinct complexes and mediate heterochromatic gene silencing by nonoverlapping mechanisms. Mol Cell 32(6): 778-790. doi: 10.1016/j.molcel.2008.10.026

\section{AUTHOR CONTRIBUTIONS}

PRG and SB designed the study. PRG generated yeast strains and performed RT-qPCR experiments with assistance by SFB. PRG and MC performed ChIP-qPCR experiments with assistance by SFB. MC performed silencing reporter assays. PRG and SB analyzed all data. SB wrote the manuscript, and PRG and MC contributed to editing.

\section{SUPPLEMENTAL MATERIAL}

All supplemental data for this article are available online at www.microbialcell.com.

\section{CONFLICT OF INTEREST}

The authors declare that they have no conflict of interest.

\section{COPYRIGHT}

(C) 2020 Georgescu et al. This is an open-access article released under the terms of the Creative Commons Attribution (CC BY) license, which allows the unrestricted use, distribution, and reproduction in any medium, provided the original author and source are acknowledged.

Please cite this article as: Paula R. Georgescu, Matías Capella, Sabine Fischer-Burkart and Sigurd Braun (2020). The euchromatic histone mark H3K36me3 preserves heterochromatin through sequestration of an acetyltransferase complex in fission yeast. Microbial Cell 7(3): 80-92. doi: 10.15698/mic2020.03.711

9. Fischer T, Cui B, Dhakshnamoorthy J, Zhou M, Rubin C, Zofall M Veenstra TD, and Grewal SIS (2009). Diverse roles of HP1 proteins in heterochromatin assembly and functions in fission yeast. Proc Natl Acad Sci USA 106(22): 8998-9003. doi: 10.1073/pnas.0813063106

10. Sugiyama T, Cam HP, Sugiyama R, Noma K-I, Zofall M, Kobayashi R, and Grewal SIS (2007). SHREC, an effector complex for heterochromatic transcriptional silencing. Cell 128(3): 491-504. doi: 10.1016/j.cell.2006.12.035

11. Job G, Brugger C, Xu T, Lowe BR, Pfister Y, Qu C, Shanker S, Baños Sanz JI, Partridge JF, and Schalch T (2016). SHREC Silences Heterochromatin via Distinct Remodeling and Deacetylation Modules. Mol Cell 62(2): 207-221. doi: 10.1016/j.molcel.2016.03.016

12. Leopold K, Stirpe A, and Schalch T (2019). Transcriptional gene silencing requires dedicated interaction between HP1 protein Chp2 and chromatin remodeler Mit1. Genes Dev 33(9-10): 565-577. doi: 10.1101/gad.320440.118

13. Verdel A, Jia S, Gerber S, Sugiyama T, Gygi S, Grewal SIS, and Moazed D (2004). RNAi-mediated targeting of heterochromatin by the RITS complex. Science 303(5658): 672-676. doi 10.1126/science.1093686

14. Martienssen R, and Moazed D (2015). RNAi and Heterochromatin Assembly. Cold Spring Harb Perspect Biol 7(8): a019323-20. doi: 10.1101/cshperspect.a019323

15. Ragunathan K, Jih G, and Moazed D (2015). Epigenetic inheritance uncoupled from sequence-specific recruitment. Science 348(6230): 1258699-1258699. doi: 10.1126/science.1258699 
16. Audergon $P$, Audergon PNCB, Catania $S$, Catania $S$, Kagansky $A$, Tong P, Shukla M, Pidoux A, Pidoux AL, and Allshire RC (2015). Epigenetics. Restricted epigenetic inheritance of H3K9 methylation. Science 348(6230): 132-135. doi: 10.1126/science.1260638

17. Zofall M, Yamanaka S, Reyes-Turcu FE, Zhang K, Rubin C, and Grewal SIS (2012). RNA elimination machinery targeting meiotic mRNAs promotes facultative heterochromatin formation. Science 335(6064): 96-100. doi: 10.1126/science.1211651

18. Ayoub N, Noma K-I, Isaac S, Kahan T, Grewal SIS, and Cohen A (2003). A novel jmjC domain protein modulates heterochromatization in fission yeast. Mol Cell Biol 23(12): 4356-4370. doi: 10.1128/mcb.23.12.4356-4370.2003

19. Zofall M, and Grewal SIS (2006). Swi6/HP1 recruits a JmjC domain protein to facilitate transcription of heterochromatic repeats. Mol Cell 22(5): 681-692. doi: 10.1016/j.molcel.2006.05.010

20. Shimada A, Dohke K, Sadaie M, Shinmyozu K, Nakayama J-I, Urano $T$, and Murakami $Y$ (2009). Phosphorylation of Swi6/HP1 regulates transcriptional gene silencing at heterochromatin. Genes Dev 23(1): 18-23. doi: 10.1101/gad.1708009

21. Barrales RR, Forn M, Georgescu PR, Sarkadi Z, and Braun S (2016). Control of heterochromatin localization and silencing by the nuclear membrane protein Lem2. Genes Dev 30(2): 133-148. doi: 10.1101/gad.271288.115

22. Kowalik KM, Shimada Y, Flury V, Stadler MB, Batki J, and Bühler M (2015). The Paf1 complex represses small-RNA-mediated epigenetic gene silencing. Nature 520(7546): 248-252. doi: 10.1038/nature14337

23. Yu R, Wang X, and Moazed D (2018). Epigenetic inheritance mediated by coupling of RNAi and histone H3K9 methylation. Nature 558(7711): 615-619. doi: 10.1038/s41586-018-0239-3

24. Shimada $Y$, Mohn F, and Bühler M (2016). The RNA-induced transcriptional silencing complex targets chromatin exclusively via interacting with nascent transcripts. Genes Dev 30(23): 2571-2580. doi: 10.1101/gad.292599.116

25. Verrier L, Taglini F, Barrales RR, Webb S, Urano T, Braun S, and Bayne EH (2015). Global regulation of heterochromatin spreading by Leo1. Open Biol 5(5): 150045-150045. doi: 10.1098/rsob.150045

26. Sadeghi L, Prasad P, Ekwall K, Cohen A, and Svensson JP (2015). The Paf1 complex factors Leo1 and Paf1 promote local histone turnover to modulate chromatin states in fission yeast. EMBO Rep 16(12): 1673-1687. doi: 10.15252/embr.201541214

27. Duempelmann L, Mohn F, Shimada Y, Oberti D, Andriollo A, Lochs $S$, and Bühler M (2019). Inheritance of a Phenotypically Neutral Epimutation Evokes Gene Silencing in Later Generations. Mol Cell 74(3): 534-541.e4. doi: 10.1016/j.molcel.2019.02.009

28. Wang $Y$, Kallgren SP, Reddy BD, Kuntz K, López-Maury L, Thompson J, Watt S, Ma C, Hou H, Shi Y, Yates JR, Bähler J, O'Connell MJ, and Jia $S$ (2012). Histone $H 3$ lysine 14 acetylation is required for activation of a DNA damage checkpoint in fission yeast. J Biol Chem 287(6): 4386-4393. doi: 10.1074/jbc.M111.329417

29. Gómez EB, Espinosa JM, and Forsburg SL (2005). Schizosaccharomyces pombe mst2+ encodes a MYST family histone acetyltransferase that negatively regulates telomere silencing. Mol Cell Biol 25(20): 8887-8903. doi: 10.1128/MCB.25.20.8887-8903.2005

30. Reddy BD, Wang Y, Niu L, Higuchi EC, Marguerat SB, Bähler J, Smith GR, and Jia $S$ (2011). Elimination of a specific histone H3K14 acetyltransferase complex bypasses the RNAi pathway to regulate pericentric heterochromatin functions. Genes Dev 25(3): 214-219. doi: 10.1101/gad.1993611
31. Flury V, Georgescu PR, lesmantavicius V, Shimada Y, Kuzdere T, Braun S, and Bühler M (2017). The Histone Acetyltransferase Mst2 Protects Active Chromatin from Epigenetic Silencing by Acetylating the Ubiquitin Ligase Brl1. Mol Cell 67(2): 294-307.e9. doi: 10.1016/j.molcel.2017.05.026

32. Gilbert TM, McDaniel SL, Byrum SD, Cades JA, Dancy BCR, Wade $H$, Tackett AJ, Strahl BD, and Taverna SD (2014). A PWWP domaincontaining protein targets the NuA3 acetyltransferase complex via histone $\mathrm{H} 3$ lysine 36 trimethylation to coordinate transcriptional elongation at coding regions. Mol Cell Proteomics 13(11): 2883-2895. doi 10.1074/mcp.M114.038224

33. Wagner EJ, and Carpenter PB (2012). Understanding the language of Lys36 methylation at histone H3. Nat Rev Mol Cell Biol 13(2): 115126. doi: $10.1038 / \mathrm{nrm} 3274$

34. Suzuki S, Kato $H$, Suzuki $Y$, Chikashige $Y$, Hiraoka $Y$, Kimura $\mathrm{H}, \mathrm{Na}$ gao K, Obuse C, Takahata S, and Murakami Y (2016). Histone H3K36 trimethylation is essential for multiple silencing mechanisms in fission yeast. Nucleic Acids Res 44(9): 4147-4162. doi: 10.1093/nar/gkw008

35. Tanny JC (2014). Chromatin modification by the RNA Polymerase II elongation complex. Transcription 5(5): e988093-10. doi: 10.4161/21541264.2014.988093

36. Govind CK, Qiu H, Ginsburg DS, Ruan C, Hofmeyer K, Hu C, Swaminathan V, Workman JL, Li B, and Hinnebusch AG (2010). Phosphorylated Pol II CTD Recruits Multiple HDACs, Including Rpd3C(S), for Methylation-Dependent Deacetylation of ORF Nucleosomes. Mol Cell 39(2): 234-246. doi: 10.1016/j.molcel.2010.07.003

37. Carrozza MJ, Li B, Florens L, Suganuma T, Swanson SK, Lee KK, Shia W-J, Anderson S, Yates J, Washburn MP, and Workman JL (2005). Histone H3 Methylation by Set2 Directs Deacetylation of Coding Regions by Rpd3S to Suppress Spurious Intragenic Transcription. Cell 123(4): 581-592. doi: 10.1016/j.cell.2005.10.023

38. Keogh M-C, Kurdistani SK, Morris SA, Ahn SH, Podolny V, Collins SR, Schuldiner M, Chin K, Punna T, Thompson NJ, Boone C, Emili A, Weissman JS, Hughes TR, Strahl BD, Grunstein M, Greenblatt JF, Buratowski S, and Krogan NJ (2005). Cotranscriptional Set2 Methylation of Histone H3 Lysine 36 Recruits a Repressive Rpd3 Complex. Cell 123(4): 593-605. doi: 10.1016/j.cell.2005.10.025

39. Joshi AA, and Struhl K (2005). Eaf3 Chromodomain Interaction with Methylated H3-K36 Links Histone Deacetylation to Pol II Elongation. Mol Cell 20(6): 971-978. doi: 10.1016/j.molcel.2005.11.021

40. Li B, Gogol M, Carey M, Lee D, Seidel C, and Workman JL (2007). Combined action of PHD and chromo domains directs the Rpd3S HDAC to transcribed chromatin. Science 316(5827): 1050-1054. doi: 10.1126/science.1139004

41. Nicolas E, Yamada T, Cam HP, Fitzgerald PC, Kobayashi R, and Grewal SIS (2007). Distinct roles of HDAC complexes in promoter silencing, antisense suppression and DNA damage protection. Nat Struct Mol Biol 14(5): 372-380. doi: 10.1038/nsmb1239

42. Creamer KM, Job G, Shanker S, Neale GA, Lin Y-C, Bartholomew B, and Partridge JF (2014). The Mi-2 homolog Mit1 actively positions nucleosomes within heterochromatin to suppress transcription. Mol Cell Biol 34(11): 2046-2061. doi: 10.1128/MCB.01609-13

43. Chen ES, Zhang K, Nicolas E, Cam HP, Zofall M, and Grewal SIS (2008). Cell cycle control of centromeric repeat transcription and heterochromatin assembly. Nature 451(7179): 734-737. doi: $10.1038 /$ nature06561

44. Matsuda A, Chikashige Y, Ding D-Q, Ohtsuki C, Mori C, Asakawa H, Kimura H, Haraguchi T, and Hiraoka Y (2015). Highly condensed chromatins are formed adjacent to subtelomeric and decondensed silent chromatin in fission yeast. Nat Commun 6(1): 7753 . doi: 10.1038/ncomms8753 
45. Cam HP, Sugiyama T, Chen ES, Chen X, Fitzgerald PC, and Grewal SIS (2005). Comprehensive analysis of heterochromatin- and RNAimediated epigenetic control of the fission yeast genome. Nat Genet 37(8): 809-819. doi: 10.1038/ng1602

46. Fleming $A B$, Kao C-F, Hillyer C, Pikaart M, and Osley MA (2008). $\mathrm{H} 2 \mathrm{~B}$ ubiquitylation plays a role in nucleosome dynamics during transcription elongation. Mol Cell 31(1): 57-66. doi: 10.1016/j.molcel.2008.04.025

47. Zofall M, and Grewal SIS (2007). HULC, a histone H2B ubiquitinating complex, modulates heterochromatin independent of histone methylation in fission yeast. J Biol Chem 282(19): 14065-14072. doi: 10.1074/jbc.M700292200

48. Bah A, Wischnewski H, Shchepachev V, and Azzalin CM (2012). The telomeric transcriptome of Schizosaccharomyces pombe. Nucleic Acids Res 40(7): 2995-3005. doi: 10.1093/nar/gkr1153

49. Greenwood J, and Cooper JP (2012). Non-coding telomeric and subtelomeric transcripts are differentially regulated by telomeric and heterochromatin assembly factors in fission yeast. Nucleic Acids Res 40(7): 2956-2963. doi: 10.1093/nar/gkr1155

50. van Emden TS, Forn M, Forne I, Sarkadi Z, Capella M, Martín Caballero L, Fischer Burkart S, Brönner C, Simonetta M, Toczyski D, Halic M, Imhof A, and Braun S (2019). Shelterin and subtelomeric DNA sequences control nucleosome maintenance and genome stability. EMBO Rep 20(1): e47181. doi: 10.15252/embr.201847181

51. Wang J, Reddy BD, and Jia S (2015). Rapid epigenetic adaptation to uncontrolled heterochromatin spreading. elife 4. doi: 10.7554/eLife.06179

52. Shi X, Kachirskaia I, Walter KL, Kuo J-HA, Lake A, Davrazou F, Chan SM, Martin DGE, Fingerman IM, Briggs SD, Howe L, Utz PJ, Kutateladze TG, Lugovskoy AA, Bedford MT, and Gozani O (2007). Proteome-wide Analysis in Saccharomyces cerevisiaeldentifies Several PHD Fingers as Novel Direct and Selective Binding Modules of Histone H3 Methylated at Either Lysine 4 or Lysine 36. J Biol Chem 282(4): 2450-2455. doi: 10.1074/jbc.C600286200

53. Grewal SI, Bonaduce MJ, and Klar AJ (1998). Histone deacetylase homologs regulate epigenetic inheritance of transcriptional silencing and chromosome segregation in fission yeast. Genetics 150(2): 563576. PMID: 9755190
54. Bao K, Shan C-M, Moresco J, Yates J III, Yates J, and Jia S (2019). Anti-silencing factor Epe1 associates with SAGA to regulate transcription within heterochromatin. Genes Dev 33(1-2): 116-126. doi: 10.1101/gad.318030.118

55. Nakayama J-I, Xiao G, Noma K-I, Malikzay A, Bjerling P, Ekwall K, Kobayashi R, and Grewal SIS (2003). Alp13, an MRG family protein, is a component of fission yeast $\mathrm{Clr} 6$ histone deacetylase required for genomic integrity. EMBO J 22(11): 2776-2787. doi: 10.1093/emboj/cdg248

56. Li B, Jackson J, Simon MD, Fleharty B, Gogol M, Seidel C, Workman $\mathrm{JL}$, and Shilatifard A (2009). Histone H3 lysine 36 dimethylation (H3K36me2) is sufficient to recruit the Rpd3s histone deacetylase complex and to repress spurious transcription. J Biol Chem 284(12): 7970-7976. doi: 10.1074/jbc.M808220200

57. Gonzalez-Sandoval A, Towbin BD, Kalck V, Cabianca DS, Gaidatzis D, Hauer MH, Geng L, Wang L, Yang T, Wang X, Zhao K, and Gasser SM (2015). Perinuclear Anchoring of H3K9-Methylated Chromatin Stabilizes Induced Cell Fate in C. elegans Embryos. Cell 163(6): 1333-1347. doi: 10.1016/j.cell.2015.10.066

58. Towbin BD, González-Aguilera C, Sack R, Gaidatzis D, Kalck V, Meister P, Askjaer P, and Gasser SM (2012). Step-wise methylation of histone H3K9 positions heterochromatin at the nuclear periphery. Cell 150(5): 934-947. doi: 10.1016/j.cell.2012.06.051

59. Cabianca DS, Muñoz-Jiménez C, nez CMXO-JX, Kalck V, Kalck VXR, Gaidatzis D, Padeken J, Seeber A, Askjaer P, and Gasser SM (2019). Active chromatin marks drive spatial sequestration of heterochromatin in C. elegans nuclei. Nature 569(7758): 734-739. doi: 10.1038/s41586-019-1243-y

60. Yu R, Jih G, Iglesias N, and Moazed D (2014). Determinants of heterochromatic siRNA biogenesis and function. Mol Cell 53(2): 262276. doi: 10.1016/j.molcel.2013.11.014

61. Hagan I, Carr AM, Grallert A, Nurse P (2016). Fission Yeast: A Laboratory Manual. Cold Spring Harbor Press, New York.

62. Braun S, Garcia JF, Rowley M, Rougemaille M, Shankar S, and Madhani HD (2011). The Cul4-Ddb1-Cdt2 ubiquitin ligase inhibits invasion of a boundary-associated antisilencing factor into heterochromatin. Cell 144(1): 41-54. doi: 10.1016/j.cell.2010.11.051 